\title{
Bronchogenic carcinoma presenting as carcinomatous meningitis
}

\begin{abstract}
Carcinomatous meningitis is defined as metastatic infiltration of cerebrospinal fluid (CSF) and leptomeninges by cancer with primary focus at some other site. This is not so common complication of lung cancer. Bronchogenic carcinoma presenting primarily with central nervous symptoms is even more uncommon. Demonstration of neoplastic cells in the CSF is key to the diagnosis. This complication impacts on the poor survival as well as poor performance status of the cancer afflicted individual. A rare case of such a manifestation is described.
\end{abstract}

Keywords: carcinomatous meningitis, bronchogenic carcinoma
Volume 4 Issue 5 - 2017

\section{Upadhyay S \\ Yashoda Superspeciality Hospital, India}

Correspondence: Sushil Upadhyay, Yashoda Superspeciality Hospital, H 104 swarn residency, I 32 GT road Sahibabad, Ghaziabad, UP, India, Tel 9899393006 , Email skupadhyay2002@rediffmail.com

Received: August 20, 2017 | Published: September 15, 2017
Abbreviations: CNF, central nervous system; CSF, cerebrospinal fluid; LMM, leptomeningeal metastasis; NSCLC, nonsmall-cell lung cancer; CT, computerised tomography; CNS, central nervous system

\section{Introduction}

Neoplastic meningitis, also known as carcinomatous meningitis, reflects the metastatic infiltration of the cerebrospinal fluid (CSF) and leptomeninges (pia and arachnoid) by cancer. It is the is the third most common cause of central nervous system (CNS) metastases after brain metastases and epidural spinal cord compression. ${ }^{1}$ Leptomeningeal metastasis (LMM) is a devastating complication of malignancy, affecting patients with both solid tumors and hematologic malignancies. The prognosis of LMM is poor, with patients reported to survive for 1-6 months from the time of the diagnosis. ${ }^{2}$ The solid tumors that most frequently give rise to LMM are breast carcinoma, melanoma, and lung carcinoma. ${ }^{3}$ The incidence of neoplastic meningitis in non-small-cell lung cancer (NSCLC) is approximately $1 \%$ to $3 \%$. Neoplastic meningitis is diagnosed in most patients with active systemic disease ( $70 \%$ overall). However, rarely it may be the first manifestation of the cancer $(5 \%){ }^{4}$

\section{Case report}

Fifty two year old Iraqi national presented with altered sensorium. He had 25 pack years of smoking history but without any symptoms or treatment related to chronic obstructive pulmonary disease. There was no other medical or surgical history as well. Physical examination revealed normal vital signs. Abdomen, cardiovascular and respiratory system were unremarkable. On neurological assessment he qualified Glassgow Coma Scale of 12/15. However there was no focal neurological deficit. Haemogram, liver and kidney biochemistries were acceptable. Computerised tomography (CT) of head did not reveal any abnormality. Lumbar puncture for cerebrospinal fluid (CSF) examination was performed. It revealed high pressure with mononuclear pleocytosis, high protein and below normal sugar. India ink preparation, smear for acid fast bacilli and gram stain were unyielding. Cytology revealed fair number atypical cells singly and in clusters, larger in size with high nucleo-cytoplasmic ratio, coarse chromatin and scanty to moderate cytoplasm with few binucleate forms. Features highly suggestive of metastasis. This lead to search for primary site of malignancy. Chest skiagram (Figure 1) of the patient showed rounded opacity in right upper zone of lung. Contrast enhanced CT scan of chest was requested and it unvield speculated mass lesion in the upper lobe of right lung (Figure 2). Videobronchoscopy examination revealed abnormal findings in the right upper lobe. Mucosa appeared swollen with rough surface but without any endobronchial growth. Rest of the bronchial tree was normal. Bronchial washings, endobronchial and transbronchial biopsies were obtained from the right upper lobe. Histopathology of biopsy was positive for non small cell carcinoma of the lung subtype adenocarcinoma (Figure 3)

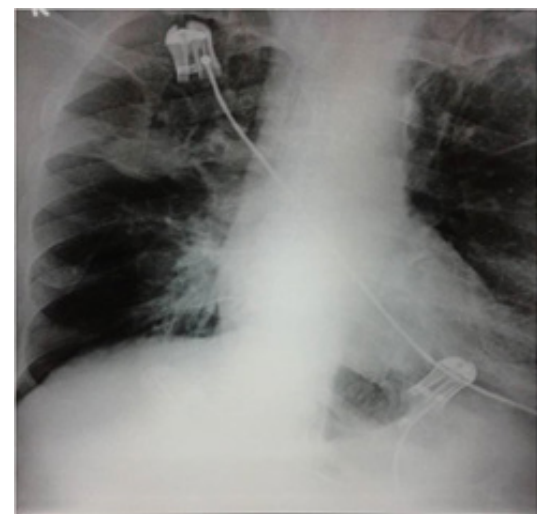

Figure I Chest Xray: Right upper lobe opacity.

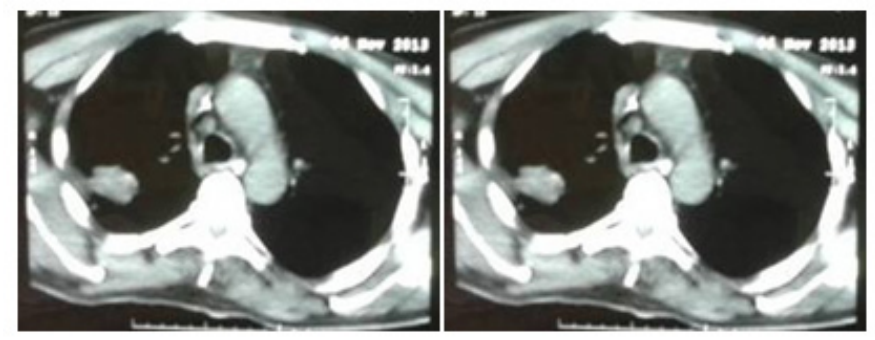

Figure 2 CT Scan of thorax: Right upper lobe mass.

\section{Discussion}

Neoplastic meningitis is the third most common metastatic complication of central nervous system (CNS) after brain metastases 
and epidural spinal cord compression. ${ }^{5}$ The diagnosis and treatment of neoplastic meningitis are of great clinical relevance due to their impact on patient quality of life and rate of survival. Such patients mostly have poor performance status with median survival of approximately two months without treatment. ${ }^{5}$ On the other hand, incidence of neoplastic meningitis is increasing due in part to improved rates of survival, because of availability of more effective systemic agents but with poor CNS penetration. ${ }^{6}$ Estimates from different studies quote leptomeningeal involvement in approximately $2-5 \%$ of patients with breast carcinoma, $9-25 \%$ of patients with small cell lung carcinoma (SCLC) and $23 \%$ of those with melanoma. ${ }^{7-9} 1-3 \%$ of non-small cell lung cancer is reported to develop carcinomatous meningitis. ${ }^{6}$

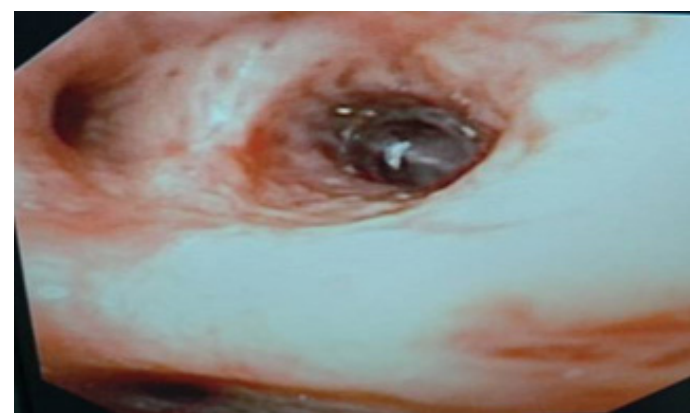

Figure 3 Bronchoscopy: Right upper lobe mucosa swollen and irregular.

The clinical presentation of neoplastic meningitis is multifocal depending on which domain of nervous system is involved. The most frequent manifestations are headache, change in mental status, gait abnormality, vomiting (cerebral hemisphere dysfunction), diplopia and facial paresis(cranial nerve involvement), lower extremity weakness and paresthesias, back or neck pain, and radiculopathy (spinal cord or exiting nerve-root manifestations). ${ }^{10}$ Meningissmus (neck stiffness) and seizures are uncommon presentation occurring in fewer than $15 \%$ and $10 \%$ of patients respectively. ${ }^{5}$ Though most patients who present with carcinomatous meningitis have a past history of treated malignancy or active malignancy, some patients have their cancer diagnosed subsequent to diagnosis of carcinomatous meningitis. ${ }^{2}$ The described case also presented primarily with neurological features.

Diagnosis of carcinomatous meningitis is established by examination of cerebrospinal fluid (CSF). Demonstration of tumor cells in CSF using flow cytometry, cytospin, centrifugation, or Millipore filtering is the gold standard test. A high opening pressure $(>200 \mathrm{~mm} \mathrm{H} \mathrm{O})$, elevated protein $(>60 \mathrm{mg} / \mathrm{dl})$, reduced glucose levels $(<50 \mathrm{mg} / \mathrm{dl})$ favour the diagnosis but are not conclusive. Specificity of CSF examination is high $(80-95 \%)$ but sensitivity is low (below $50 \%$ ). Repeat lumbar puncture(usually two attempts), generous volume $(>10 \mathrm{ml})$, obtaining sample from involved site, avoiding hemorrhagic sample and prompt processing increases the yield. ${ }^{1,5,10}$ MRI with gadolinium enhancement is recommended ideally before the lumbar puncture to localize the disease sites in the neuraxis as indicated by abnormal contrast enhancement. MRI shows uptake in up to $40-60 \%$ of patients. ${ }^{11}$ Whereas CT scan of cranium has limited value (sensitivity $15-25 \%$ ) in detecting neoplastic meningitis sensitivity of MRI is $40-80 \% .{ }^{5,12}$ Findings on MRI scan include focal or diffuse pial enhancement; ependymal, sulcal, folia, or cranial nerve enhancement; and subarachnoid, ventricular, or parenchymal-enhancing nodules. Hydrocephalus, which is a secondary consequence of neoplastic meningitis, may also be observed. ${ }^{4}$
Management of carcinomatous meningitis is multimodal and includes surgery (placement of intraventricular reservoir and ventriculo-peritoneal shunt), radiation at high burden site and chemotherapy both systemic and intrathecal. As overall outcome is poor, decision to treat needs careful case selection. Patients with good performance status and lesser disease burden usually benefit from treatment. Several studies have suggested that treatment does improve quality of life and prolongs survival. ${ }^{9,13}$

\section{Conclusion}

Carcinomatous meningitis is a rare but serious complication of lung cancer. It poses a real diagnostic challenge to clinician when other systemic cancers present primarily with CNS symptoms. Prognosis remains poor and there is little improvement in quality of life and survival with treatment.

\section{Acknowledgements}

None.

\section{Conflicts of interest}

The authors declare that there is no conflict of interests regarding the publication of this paper.

\section{Funding}

None.

\section{References}

1. Chamberlain M, Soffietti R, Raizer J, et al. Leptomeningeal metastasis: a response assessment in neuro-oncology critical review of endpoints and response criteria of published randomized clinical trials. Neuro Oncol. 2014;16(9):1176-1185.

2. Balm M, Hammack J. Leptomeningeal carcinomatosis. Presenting features and prognostic factors. Arch Neurol. 1996 53(7):626-632.

3. Bleyer WA, Byrne TN. Leptomeningeal cancer in leukemia and solid tumors. Curr Probl Cancer. 1996;12(4):181-238.

4. Le Rhun E, Taillibert S, Chamberlain MC. Carcinomatous meningitis: leptomeningeal metastases in solid tumors. Surg Neurol In. 2013;4(Suppl 4):S265-S288.

5. Chamberlain MC. Leptomeningeal metastasis. Curr Opin Neurol. 2009;22(6):665-674.

6. Omuro AM, Kris MG, Miller VA, et al. High incidence of disease recurrence in the brain and leptomeninges in patients with nonsmall cell lung carcinoma after response to gefitinib. Cancer. 2005;103(11):23442348

7. Yap HY, Yap BS, Tashima CK, et al. Meningeal carcinomatosis in breast cancer. Cancer. 1978;42(1):283-286.

8. Aroney RS, Dalley DN, Chan WK, et al. Meningeal carcinomatosis in small cell carcinoma of the lung. Am J Med. 1981;71(1):26-32.

9. Amer MH, Al-Sarraf M, Baker LH, et al. Malignant melanoma and central nervous system metastases: incidence, diagnosis, treatment and survival. Cancer. 1978;42(2):660-668.

10. Little JR, Dale AJ, Okazaki H. Meningeal carcinomatosis. Clinical manifestations. Arch Neurol. 1974;30(2):138-143.

11. Glantz MJ, Cole BF, Glantz LK, et al. Cerebrospinal fluid cytology in patients with cancer: minimizing false-negative results. Cancer 1998;82(43):733-739. 
12. Chamberlain MC. Comprehensive neuraxis imaging in leptomeningeal metastasis: a retrospective case series. CNS Oncol. 2013;2(2):121-128.
13. Park JH, Kim YJ, Lee JO, et al. Clinical outcomes of leptomeningeal metastasis in patients with non-small cell lung cancer in the modern chemotherapy era. Lung Cancer. 2012;76(3):387-392. 